\title{
Hydroxysafflor yellow A promotes osteogenesis and bone development via epigenetically regulating $\beta$ - catenin and prevents ovariectomy-induced bone loss
}

\section{Peng Wang}

Guangzhou University of Chinese Medicine

\section{Tingling Zhuo}

Guangzhou University of Chinese Medicine

\section{Ying Li}

Guangzhou University of Chinese Medicine

\section{Meng Zhang}

Guangzhou University of Chinese Medicine

\section{Chi Zhou}

Guangzhou University of Traditional Chinese Medicine First Affiliated Hospital Jinfang Zhang

Guangzhou University of Chinese Medicine

\section{Gang Li}

Chinese University of Hong Kong

\section{Haibin Wang}

Guangzhou University of Traditional Chinese Medicine First Affiliated Hospital

Liangliang Xu ( $\nabla$ xull-2016@gzucm.edu.cn)

Guangzhou University of Chinese Medicine https://orcid.org/0000-0002-5249-7480

\section{Research}

Keywords: Hydroxysafflor yellow A, Osteoporosis, Histone demethylase, $\beta$-catenin

Posted Date: July 1st, 2020

DOl: https://doi.org/10.21203/rs.3.rs-38205/v1

License: (c) (1) This work is licensed under a Creative Commons Attribution 4.0 International License. Read Full License 
Version of Record: A version of this preprint was published at The International Journal of Biochemistry \& Cell Biology on August 1st, 2021. See the published version at https://doi.org/10.1016/j.biocel.2021.106033. 


\section{Abstract}

Objectives: Mesenchymal stem cells (MSCs) forming excessive adipocytes has been proven to be the pathogenesis of osteoporosis, leaving the bone more fragile. The approaches to promoting osteogenesis of MSCs are regarded as the promising direction to prevent osteoporosis. In clinical treatment, there is increasingly prevalent that traditional Chinese medicine treats common bone diseases including osteoporosis. HuoXue Tong Luo capsule (HXTL capsule) containing Safflower, Angelica sinensisetc, is one of the most frequently used prescriptions for osteoporosis with promising effects. In our study, we have demonstrated Hydroxysafflor yellow A (HSYA), belonging to one of essential compounds of Safflower, promote bone development and inhibit ovariectomized osteoporosis and explored its potential mechanisms in vitro.

Materials and methods: We first investigated the effects of HSYA on viability and osteogenesis in human bone marrow-derived mesenchymal stem cells (hBMSCs). RNA sequencing analysis and ChIP assay were conducted to find out the potential mechanism of bone formation effect of HSYA. In vivo, through the chick embryo and ovariectomy (OVX)-induced osteoporosis rat model, we analyzed the function of bone development and formation of HSYA.

Results: Taken all the results of our experiments together, it is safe to conclude that HSYA can promote osteogenesis via KDM7A as an epigenetic role to activate histone demethylation in $\beta$-catenin. More importantly, our study indicated that HSYA could promote bone development and protect against OVXinduced bone loss.

Conclusion: These findings could pave the way to the potential development of HSYA-targeted therapeutic treatments for skeletal diseases such as osteoporosis.

\section{Introduction}

Bone is a complex tissue which protects our various soft tissues and maintains the mineral metabolism in the microenvironment [1]. In vertebrates, the long bone development starts through Cartilaginous Internalized Bone when MSCs proliferate and densify into osteoblasts that secrete the osteoid and turn into bone cells [2]. MSCs are capable of self-replication and have multiple differentiation potential, including the osteoblast and the adipocyte. Osteogenesis and adipogenesis of MSCs are strictly regulated under various physiological signal transduction pathways to maintain dynamic balance. The ability to form adipocytes of MSCs will be inhibited when MSCs differentiate toward osteoblasts, and vice versa [3]. Therefore, excessive adipocytes have been proven to be the pathogenesis of several bone disorders, including osteoporosis [4], which may be related to bone loss.

Wnt/ $\beta$-catenin, TGF- $\beta$ /BMPs/Smads pathways play the key roles in osteogenic differentiation of MSCs. $\beta$-catenin, Runx2 and PPARy are important transcriptions factors for MSCs to differentiate into osteoblasts and adipocytes [5]. Wnt/ $\beta$-catenin pathway is reported that it can mediate more than 50 type's proteins which are able to stimulate biological responses of various cells in regulating the internal 
environment of human body. The key switch in the canonical Wnt pathway is the cytoplasmic protein $\beta$ catenin [6]. Wnt protein acts on $\beta$-catenin's cell-surface receptor to prevent $\beta$-catenin degradation. Then, the up-regulated $\beta$-catenin can translocate into the nucleus and stimulate the expression of different down-stream genes. $\beta$-catenin is required for MSCs to differentiate into either osteoblasts or chondrocytes during the early stages of fracture repair $[7,8]$. The researchers created a type of mice with $\beta$-catenin depletion in the osteoblast lineage through using alpha 1 type 1 collage-Cre (Col1a1-Cre) mice. They found that the mice with $\beta$-catenin depletion present a low bone mass phenotype because of increased osteoclastic bone resorption due to decreased expression of osteoprotegerin (OPG) [9].

In orthopaedic diseases, multiple experimental studies have shown that epigenetic factors are involved in the process of bone growth, regeneration and repair. This process includes the dynamic balance of osteoblast and adipocyte regulation. Our previous multiple studies have demonstrated that epigenetic regulation such as DNA methylation and histone methylation are involved in many aspects such as differentiation and migration of bone marrow-derived mesenchymal stem cells (BMSCs) [10], and the incidence of osteoporosis [11]. In addition, we found Huo Xue Tong Luo capsule, which can promote blood circulation and remove blood stasis, with nourishing kidney and bone in Chinese medicine, significantly increased the binding of H3K27me3 in the IncRNA-Miat promoter region and reduced the binding of $\mathrm{H} 3 \mathrm{~K} 4 \mathrm{me} 3$, indicating that this capsule can inhibit directly the expression of Miat through the histone modification [12]. Also, there is the specific connection between $\beta$-catenin and epigenetics in osteogenesis. MiR-29a can regulate the acetylation of $\beta$-catenin, which directly influences the activities of osteoblast cultures [13]. Histone methyltransferase SETD2 regulates osteosarcoma cell growth and chemosensitivity by suppressing Wnt/ $\beta$-catenin signaling [14]. Knocking-down $\beta$-catenin reduced EZH2 (a key component of the PRC2 complex that catalyzes histone methylation) protein levels and H3K27me3 at osteogenic loci [15]. Our previous research showed that IncRNA H19 can promote osteoblast differentiation by regulating Wnt/ $\beta$-catenin pathway through acting as a competing endogenous RNA in hMSCs [16].

Safflower, is known as Carthamus tinctorius $L$, which has already been applied in various medical fileds such as digestology, anesthesiology and infectious immunology for antipyretic substances and purgative, analgesic [17]. In clinical treatment, Huo Xue Tong Luo capsule (HXTL capsule) containing Safflower, Angelica sinensis etc, is one of the mostly used prescriptions for treating osteoporosis with promising effects. Hydroxysafflor yellow A is an active compound isolated from Safflower and has been widely used as the therapy for anti-platelet and anti-myocardial ischemia, anti-inflammatory activity [18]. It is also reported that HSYA can enhance the formation of E-cadherin/ $\beta$-catenin complex for inhibiting adhesion, invasion, migration and lung metastasis of hepatoma cells [19].

Given the significant role of mesenchymal stem cells in osteoporosis, and the anti-inflammatory, antiaggregation and other applications of HSYA, we hypothesized that HSYA might promote bone development and osteogenesis. In the present research, we focused on the potential therapeutic effects of HSYA on osteogenesis of hBMSCs in vitro and the chick embryo and ovariectomy (OVX)-induced 
osteoporosis rat model in vivo. Meanwhile, through RNA-sequencing and CHIP assays we evaluated the effect of HSYA on histone demethylation to elucidate the underlying mechanisms.

\section{Materials And Methods}

\section{Chemicals and materials}

HSYA (purity $>98 \%$ by HPLC; Fig. 1a) was purchased from Weikeqi Biological Technology Corporation (Sichuan, China). Fertilized Leghorn eggs were obtained from the Avian Farm of the South China Agriculture University (Guangzhou, China). Modified Eagle's Medium of Alpha (a-MEM), fetal bovine serum (FBS) and penicillin/streptomycin were purchased from Gibco (USA). B-glycerolphosphate, dexamethasone, and ascorbic acid phosphate were purchased from Sigma (USA). Alizarin RED S was purchased from Solarbio (Beijing, China). Cell Counting Kit-8 (CCK-8) and BCIP/NBT alkaline phosphatase coloration kit were purchased from Beyotime (Beijing, China). The siRNAs targeting human KDM7A were designed and synthesized by Genepharma Company (Shanghai, China). Lipofectamine 3000 transfection reagent was obtained from Thermo (USA). Takara Mini BEST Universal RNA Extraction Kit, Prime Script RT Master Mix (Perfect Real Time) and SYBR-Green Master Mix were supplied by Takara (Japan). Trizol reagent was obtained from Invitrogen (USA). Polyvinylidene difluoride (PVDF) membrane was obtained from Millipore (USA). Primary antibody against Runx2, H3K9me2 and DAPI were supplied by Cell Signaling Technology (USA); Primary antibody against $\beta$-catenin and anti-Bglap were purchased from Bioworld (USA); Anti-Osterix, anti-Col1a1, anti-GAPDH, and anti-H3K27me2 antibodies were obtained from Abcam (USA). Secondary antibodies (HRP-conjugated Goat Anti-Rabbit IgG) were obtained from Abcam (USA). ChIP-Grade Protein G Magnetic Beads were donated from Cell Signaling (USA). DualLuciferase Reporter Assay System was supplied by Promega Company (USA).

\section{Reagents and cell culture}

Human bone marrow-derived MSCs (hBMSCs) were donated from the Cyagen Biosciences Corporation (USA) in the Guangzhou, China. The cells were kept in a-MEM supplemented 10\% FBS and 1\% penicillin/streptomycin. The surface antigens of hBMSCs clusters were detected through flow cytometry by the biological company. BMSCs between passage 2 and 8 were used in this study.

\section{Cell viability assay}

The effect of HSYA on the proliferation of hBMSCs was assessed using CCK-8 kit. In brief, an initial density of $5 \times 103$ cells/well were prepared in 96-well plates and cultured in a graded series of HSYA for 1 ,

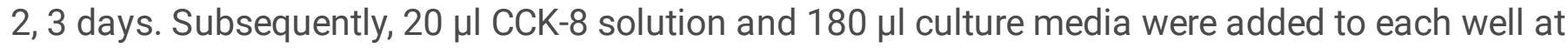
respective time point and plates were incubated for $4 \mathrm{~h}$ at $37^{\circ} \mathrm{C} .100 \mu \mathrm{l}$ aliquots were taken from each well and transferred to another 96 -well plate for final analysis. The absorbance of samples was measured at $450 \mathrm{~nm}$ with a spectrophotometric micro plate reader (Xianke Instruments, Shanghai, China). 


\section{Osteogenic induction assay and ALP staining}

To induce osteogenic differentiation, the culture media were replaced with osteogenic induction medium when cells reached a confluence of over $70 \%$. The media were changed every 2 day. The hBMSCs were treated with or without OIM containing a-MEM supplemented with $20 \mathrm{mM}$ B-glycerophosphate, $100 \mathrm{nM}$ dexamethasone, and $50 \mu \mathrm{M}$ ascorbic acid phosphate and HSYA for 7 days before the cells were washed with PBS twice and fixed with $70 \%$ ethanol for $10 \mathrm{~min}$. The cells were incubated with BCIP/NBT alkaline phosphatase coloration kit at $37^{\circ} \mathrm{C}$ for $1 \mathrm{~h}$. Then the reaction was stopped by distilled water and the plate was dried before taking photo.

\section{Mineralization assay}

After 14 days of osteogenic induction, cells were fixed with $70 \%$ ethanol for $10 \mathrm{~min}$. Then the fixed cells were stained with $0.5 \%$ alizarin red $\mathrm{S}(\mathrm{pH} 4.1)$ for $10 \mathrm{~min}$ at room temperature and washed three times with deionized water. Orange red staining indicated the position and intensity of calcium deposits.

\section{Cell transfection}

The sequence of each siRNA targeting human KDM7A was listed in Table 1. SiRNA transfection was performed with Lipofectamine 3000 transfection reagent according to the manufacturer's instructions. Briefly, hBMSCs were plated in a-MEM/OIM medium into 12-well plate, and allowed to reach $50-60 \%$ confluency. 7 days later the total RNA was extracted for real time PCR analysis.

Table 1

Sequences of siRNA targeting KDM7A.

\begin{tabular}{|c|c|c|}
\hline Gene & Sense Primer Sequence $\left(5^{\prime}-3^{\prime}\right)$ & Antisense Primer Sequence $\left(5^{\prime}-3^{\prime}\right)$ \\
\hline siKDM7A-1 & CAAGUGCCGAUGAAAUAAUTT & AUUAUUUCAUCGGCACUUGTT \\
\hline siKDM7A-2 & GCCUCAAACUUACCUAGUATT & UACUAGGUAAGUUUGAGGCTT \\
\hline siKDM7A-3 & CCAGAAGCCAUCUAGAAGUTT & ACUUCUAGAUGGCUUCUGGTT \\
\hline
\end{tabular}

Total RNA was extracted from cultured hBMSCs using Takara Mini BEST Universal RNA Extraction Kit according to the manufacturer's instructions. Briefly, the cells were lysed with Buffer RL reagent for 10 min. DNase I was used to remove contaminating DNA in total RNA. The first-strand cDNA was synthesized using Prime Script RT Master Mix (Perfect Real Time). Real-time PCR was performed using the CFX96 Real-Time PCR Detection System (Bio-Rad, USA). The reaction conditions consisted of 15 ul reaction volumes with diluted cDNA template $2 \mu \mathrm{l}, 7.5 \mu$ l SYBR-Green Master Mix (2x), 4.5 ul PCR-Grade water and $0.5 \mathrm{ul}$ of each primer $(10 \mu \mathrm{M})$. Amplification conditions were as follows: first at $95^{\circ} \mathrm{C}$ for $5 \mathrm{~min}$, and then 45 cycles of $95^{\circ} \mathrm{C}$ for $15 \mathrm{~s}$ and $60^{\circ} \mathrm{C}$ for $60 \mathrm{~s}$. Primer sequences were shown in Table 2 . The relative quantification of gene expression was normalized to the expression level of $\beta$-Actin. 
Table 2

List of primer sequences for real-time quantitative RT-PCR

\begin{tabular}{|c|c|c|}
\hline Primer name & Sense Primer Sequence $\left(5^{\prime}-3^{\prime}\right)$ & Antisense Primer Sequence $\left(5^{\prime}-3^{\prime}\right)$ \\
\hline ALP & СТСССАGTСTСАТСТССТ & AAGACСTСААСТССССТGAА \\
\hline OPG & AGGACATTTGTCACACAACAGC & GGGGACCACAATGAACAAGT \\
\hline Runx2 & АCTTCCTGTGCTCGGTGCT & GACGGTTATGGTCAAGGTGAA \\
\hline Col1a1 & CACTGGTGATGCTGGTCCTG & CGAGGTCACGGTCACGAAC \\
\hline OPN & GTACCCTGATGCTACAGACG & TTCATAАСTGTССТTCССАС \\
\hline KDMЗА & AGTGGCCCAGTGGTTGAAAA & TTGTCCCGATGATCTCCCCT \\
\hline KDM1B & GCAGTTAGAGAACATGGAGACTTTT & ATCTTGAGACAGGCGGAACC \\
\hline KDM5A & GGCAACAAGACCGTGACCTA & CCGATTGGTAATCCTGGGGG \\
\hline KDM8 & ACGCTAGTGCAGGTGTCAAAT & TTACGCTAAGCCGTTCAGCA \\
\hline KDM4A & ACCGCAAGCTCTCCAAGATT & AGATCTGTTTGGGCTGGGTG \\
\hline KDM7A & TCCAATTAAAAGGGAATGTCCTACC & CAGGAGGAAGTCCTCAAGTCT \\
\hline$\beta$-catenin & ATTGTCCACGCTGGATTTTC & AGGTCTGAGGAGCAGCTTCA \\
\hline$\beta$-Actin & CCTTGCACATGCCGGAG & GCACAGAGCCTCGCCTT \\
\hline
\end{tabular}

\section{Western blotting}

Equal proteins were loaded onto $8 \%$ SDS-PAGE and subsequently transferred onto a PVDF membrane for $120 \mathrm{~min}$ at $350 \mathrm{~mA}$. The membrane was blocked with $5 \%$ skim milk for $1 \mathrm{~h}$ at room temperature. After washing 3 times with TBS containing $0.1 \%$ Tween 20 (TBST), the membranes were incubated, respectively, with anti-Runx2 (1:1000), anti- $\beta$-catenin (1:1000), anti-Osterix (1:1000), anti-Col1a1 (1:1000) or anti-GAPDH (1:1000) antibodies at $4{ }^{\circ} \mathrm{C}$ overnight. After washing in TBST for 3 times (5 min for each time), the membrane was incubated with horseradish peroxidase-linked secondary antibodies (antirabbit) for $2 \mathrm{~h}$ at room temperature. After washing five times with TBST, proteins were detected with ECL western blotting detection reagent (Beyotime, China) according to the manufacturer's instruction.

\section{RNA sequencing analysis}

hBMSCs HSYA-treated or not at passage 5 were used for RNA sequencing (RNA-seq) analysis. The cells were washed with PBS 3 times and collected by TRIzol Reagent. The quality and integrity of total RNA samples were assessed using a 2100 Bioanalyzer or a 2200 TapeStation (Agilent Technologies) according to the manufacturer's instructions. The preparation of whole transcriptome libraries and deep sequencing were performed by the Annoroad Gene Technology Corporation (Beijing, China). DAVID and KOBAS bioinformatics tools were used for functional annotation enrichment and clustering. 
Experiments were conducted as mentioned before [20]. In brief, HEK293 cells were seeded on a 24-well plate, and the cells were allowed to grow until $80 \%$ confluency. Cells were then transfected with TOPflash (500 ng) and Renilla reporter plasmid pRL-CMV (100 ng) by using Lipofectamine 3000. Twenty-four hours after transfection, cells were treated with HSYA in different concentrations for 12 hour. The luciferase activity was measured using GloMax $^{\mathrm{TM}}$ 20/20 Single tube luminometer (Promega, Madison, WI, USA).

\section{Cell immunofluorescence}

hBMSCs were collected after $48 \mathrm{~h}$ of $10 \mu \mathrm{M}$ treatment, and inoculated into 24 -well plates with $5 \times 103$ cells $/ \mathrm{ml}$, getting ready for slide climbing. After 4 hours, the cells were fixed with $4 \%$ paraformaldehyde for

$30 \mathrm{~min} .5 \% \mathrm{BSA}$ was added to the slides and sealed at room temperature for $30 \mathrm{~min}$. $\beta$-catenin antibody (1:200)/H3K27me2 antibody (1:3200)/H3K9me2 antibody (1:200) were added to the sealant and incubated overnight at $4{ }^{\circ} \mathrm{C}$. After drying the excess liquid on the slides, fluorescent secondary antibody (1:1000) was added and incubated in the wet box at room temperature for $1 \mathrm{~h}$. DAPI was used to dye the core, and the cover glass was used to seal the sample for development and photography.

\section{Chromatin immunoprecipitation assay}

Cross-linking and chromatin immunoprecipitation (ChIP) were done as described previously [21]. Briefly, all of the subsequent steps were performed at $4{ }^{\circ} \mathrm{C}$, and all of the buffers contained $0.1 \mathrm{mM}$ EDTA, $0.5 \mathrm{mM}$ EGTA, $1 \mathrm{mM}$ dithiothreitol, and protease inhibitors (BD). The hBMSCs were washed with phosphate-buffered saline ( $\mathrm{pH} 7.4)$ and lysed. After centrifugation, the pellet was resuspended in $10 \mathrm{ml}$ of $10 \mathrm{mM}$ Tris- $\mathrm{HCl}, \mathrm{pH} 8.0$, and $200 \mathrm{mM} \mathrm{NaCl}$; centrifuged at 15,000 g for $15 \mathrm{~min}$. The chromatin pellet was resuspended in $1 \mathrm{ml}$ of $50 \mathrm{mM}$ Tris- $\mathrm{HCl}, \mathrm{pH} 7.9$, and $5 \mathrm{mM} \mathrm{CaCl} 2$ and digested with 500 units of micro coccal nuclease (New England Biolabs) at $37^{\circ} \mathrm{C}$ for $10 \mathrm{~min}$. For ChIP reactions, the samples (1 ml) were immunoprecipitated overnight with anti-H3K9me2, H3K27me2, or control rabbit IgG. ChIP-PCR analysis was done by using $3 \mu \mathrm{l}$ of ChIP DNA and primer sets shown in Table 3. For histone and $\beta$-catenin ChIPs from hBMSC cells, enrichment was determined relative to a control ChIP with IgG antibody.

Table 3

List of primer sequence for CHIP-PCR

\begin{tabular}{|lll|}
\hline Primer name & Sense Primer Sequence $\left(\mathbf{5}^{\prime} \mathbf{3}^{\prime}\right)$ & Antisense Primer Sequence $\left(\mathbf{5}^{\prime} \mathbf{3}^{\prime} \mathbf{)}\right.$ \\
\hline B-catenin-1 & ATTAGCAAAGAATCACCC & TGAACAAACCAAAGACAG \\
\hline
\end{tabular}

\section{Embryo manipulation}

Fertilized Leghorn eggs were incubated in a humidified incubator (Jinweng Instruments, Shanghai, China) at $37^{\circ} \mathrm{C}$ until the embryos reached the desired developmental stage [22]. The chick embryos were exposed to HSYA $(10 \mu \mathrm{M})$ or control DMSO in equal volume for 14 days. In brief, $200 \mu \mathrm{l} 0.9 \%$ NS including DMSO or $10 \mu \mathrm{M}$ HSYA were carefully injected into a small hole made in the air chamber of the egg on 2 day of incubation. After the treatment, the embryos were further incubated for 14 days before being harvested for analysis. 


\section{Alcian blue and alizarin red staining}

To visualize the skeleton, the chick embryos were stained with alcian blue and alizarin red dyes as described [23]. Fifteen-day-old chick embryos were fixed in $95 \%$ ethanol for 2 day with the fur and skin and viscera carefully removed, and then the specimen were post-fixed in the mixture solution $(100 \%$ ethanol/acetic acid/saturated alcian blue in 75\% ethanol, 20:5:3) for two days. The embryos were stained in saturated alizarin red (Solarbio, Beijing, China) in ultrapure water / 1\% KOH dyes for 1 week and then in $25 \%$ glycerol / $1 \% \mathrm{KOH}$ for clearing lasting 2 days. Finally, the embryos were treated in a graded series (50\%-75\%-100\%) of glycerol for 5 days respectively. To make parts of the skeleton more visible, we carefully dismembered long bone tissues and photographed them using a stereomicroscope (Ckx41 Olympus, German). The length of the alizarin red stained portion of each spine, radius, and metatarsus were quantified and analyzed using the Image $\mathrm{J}$ software.

\section{Ovariectomy (OVX)-induced osteoporosis rat model}

Twelve SD rats (females; $280-320 \mathrm{~g}, 16$ weeks old) were supplied by the Animal Experiment Center of Guangzhou University of Chinese Medicine. All rats were randomly divided into four groups: sham group $(n=3)$, OVX group $(n=3)$, OVX + HSYA $(2.5 \mathrm{mg} / \mathrm{kg})$ group $(n=3)$ and OVX + HSYA $(10 \mathrm{mg} / \mathrm{kg})$ group $(n=$ 3). Bilateral ovariectomies were performed to induce osteoporosis under chloral hydrate anesthesia for $\mathrm{OVX}$ and OVX + HSYA groups. For sham group, the ovaries were only exteriorized but not resected. All rats had 3 days recovery after the operations, and then an intraperitoneal injection of HSYA

$(2.5 \mathrm{mg} / \mathrm{kg} \& 10 \mathrm{mg} / \mathrm{kg}$, every 2-3 days for 12 weeks) was delivered for OVX + HSYA group. The sham and OVX group mice were intraperitoneally injected with $0.9 \% \mathrm{NS}$ as a vehicle control.

\section{Micro-CT analysis}

After sacrificing the experimental rat groups, right tibia ( $\mathrm{n}=3$ for each treatment group) were fixed with $4 \%$ paraformaldehyde (PFA) for 24 hours and placed in $5 \mathrm{ml}$ centrifuge tubes and scanned using Skyscan 1176 micro-CT scanner (Bruker micro-CT, Kontich, Belgium). The scanning was carried out using following settings: voltage, $50 \mathrm{kV}$; source current, $500 \mu \mathrm{A}$; Al $0.5 \mathrm{~mm}$ filter; pixel size $27 \mu \mathrm{m}$; rotation step, 0.4 degree. Cancellous bone of the proximal tibia $2.7 \mathrm{~mm}$ above the growth plate was chosen as the VOI, which was restricted to an internal region of femur where trabecular and cortical bones were extracted by drawing free form contours with the CT analyzer software. The bone volume/tissue volume (BV/TV), mean volumetric bone mineral density (BMD), trabecular number (Tb. N), trabecular thickness (Tb. Th) and trabecular separation ( $\mathrm{Tb} . \mathrm{Sp}$ ) were measured. Twoand three-dimensional images were generated using Data-viewer and CTvol softwares (Bruker micro-CT, Kontich, Belgium) respectively.

\section{Histological and immunohistochemical analyses}

These samples were washed in PBS, fixed in 4\% paraformaldehyde, decalcified, dehydrated, and embedded in paraffin. The sections were cut at a thickness of $5 \mu \mathrm{m}$ and stained with hematoxylin and eosin (H\&E) after deparaffination. As for immunohistochemical (IHC) analysis, primary antibody against Bglap protein (1:200), and goat anti-rabbit IgG horseradish peroxidase (HRP)-conjugated secondary 
antibody were used for detection. Images were acquired with the Aperio ScanScope (Leica Biosystem, Buffalo Grove, IL, USA).

\section{Statistical analysis.}

All data and statistical analysis were followed with the recommendation of pharmacology experimental design. All quantitative data was analyzed by a commercially available statistical program SPSS version 20.0 (IBM, USA). The two-tailed Student's t-test was applied for comparisons of two groups and one-way analysis of variance (ANOVA) with the Tukey' post hoc test for three or more groups. $\mathrm{P}<0.05$ as considered statistical significance. All data were presented as mean \pm SD.

\section{Results}

\section{HSYA had no effect on hBMSCs viability}

To investigate the effect of HSYA on cell viability, the CCK-8 assay was performed. The result showed that HSYA did not influence cell viability when it was used at concentration between 1 and $40 \mu \mathrm{M}$ (Fig. 1b).

\section{HSYA activated the Wnt/ $\beta$-catenin signalling pathway to promote osteogenesis of hBMSCs}

We measured the ALP activity of hBMSCs after they were incubated with different concentrations of HSYA for 7 days. ALP staining proved HSYA increased the ALP activity of hBMSCs in a dose-dependent manner (Fig. 2a). The similar results were observed by Alizarin Red S staining (Fig. 2b). The result obtained from the western blotting demonstrated that the protein level of Runx2 is up-regulated by HSYA in different concentrations (Fig. 2c). Furthermore, we detected the gene expression of essential osteogenic markers, and transcription Runx2. The real time PCR result showed that OPN, Runx2 and Col1a1 were significantly up-regulated by HSYA at $10 \mu \mathrm{M}$ (Fig. 2d). It is well known that Wnt/ $\beta$-catenin signaling pathway is one of the most important signaling pathways, playing an important role in regulation of osteogenic differentiation of BMSCs. In order to check whether Wnt/ $\beta$-catenin signaling pathway was activated or not when hBMSCs were treated with HSYA, we performed western blotting to detect the level of $\beta$-catenin in hBMSCs, and the result showed that $\beta$-catenin was significantly upregulatedin hBMSCs when they were treated with OIM (Fig. 2e). Also, the effect of HSYA on the luciferase activity of Wnt signaling reporter TOPflash was investigated. After 12-hour stimulation, the luciferase activities were measured at different concentrations with the highest one at concentration of $10 \mu \mathrm{M}$ (Fig. 2f). More importantly, it is reported the successful nucleus translocation of $\beta$-catenin is directly associated with the activation of the signaling pathway, and then immunolabeling and fluorescence microscopy of $\beta$-catenin were undertaken. The result demonstrated that less expression of $\beta$-catenin (stained with red color) in the nucleus of the hBMSCs (stained with blue color) was observed in the Ctrl. Through adding HSYA $(10 \mu \mathrm{M})$ into hBMSCs, enhanced translocation of $\beta$-catenin was noticed in the nucleus (Fig. 2g). 


\section{Identification of HSYA-responsive mRNA in hBMSCs}

RNA sequencing analysis is an effective method for the prediction of the mechanisms underlying the effects of HSYA. The expression of detected mRNAs of HSYA-treated hBMSCs vs control hBMSCs are shown in supplementary file 1. The heatmap and volcano map were shown in (Fig. 3a-b). The Kyoto Encyclopedia of Genes and Genomes (KEGG) analysis revealed that several signaling pathways were enriched as shown in supplementary file 2. From the results of RNA sequencing analysis, we chose six KDMs orthologous genes with differential expression, coding their namesake proteins with the histone demethylase signature domain Jumonji $C$ (JmjC), for further verification. We found the most conspicuous one was KDM7A through real time PCR, as its expression was significantly up-regulated in HSYA groups (Fig. 3c). The function of KDM7A in osteogenesis of hBMSCs is largely unknown, so further invitro analyses of KDM7A were conducted.

\section{HSYA positively regulated osteogenesis of hBMSCs via KDM7A}

To evaluate the role of KDM7A in osteogenesis of hBMSCs, we transfected hBMSCs with negative control, siRNA-KDM7A respectively. Three siRNAs were transfected into hBMSCs and we found that siRNA-KDM7A-2 significantly decreased the expression of KDM7A mRNA (Fig. 4a). So we chose siRNAKDM7A-2 for further experiment. The Alizarin Red S staining and western blotting results revealed that the osteogenesis of hBMSCs significantly decreased in the siRNA-KDM7A group, compared with the NC group (Fig. $4 \mathrm{~b}$-c). Furthermore, this decline tendency because of the depletion of KDM7A can be inverted partly which were proved in real-time PCR and western blotting results (Fig. 4d-e).

\section{HSYA promoted $\beta$-catenin expression in hMSCs by histone modifications}

For exploring whether the KDM7A plays an epigenetic role in mechanism of osteogenesis effect of HSYA, we tested the expression level of di-methylation marks H3K9m2 (repressive histone modification) and H3K27m2 (repressive histone modification) after HSYA interference in hBMSCs by conducting the cell immunofluorescence. The fluorescin could be identified in cell nucleus and the HSYA-treated groups showed the darker brightness respectively (Fig. 5a-b). Furthermore, we checked the H3K9m2 and H3K27me2 occupancies in the region of the $\beta$-catenin's promoter of hBMSC treated by HSYA $(10 \mu M)$, as the histone methylations are associated with target gene expression in stem cells to regulate cell fate. The chromatin immunoprecipitation (ChIP) assay demonstrated the occupancy of H3K27me2 on the promoter region of $\beta$-catenin was significantly decreased, but the ratio of binding of $\mathrm{H} 3 \mathrm{~K} 9 \mathrm{me} 2$ was not distinctly different comparing with that of control group (Fig. 5c). The results showed HSYA is likely to be capable of regulating bone formation via epigenetic impact of KDM7A targeting di-methylation marks H3K27m2. 


\section{HSYA exposure accelerated endochondral ossification during embryo development}

According to above results, we conducted the chick embryo model to evaluate whether HSYA can accelerate bone development of vertebrates. The spine, radius, and metatarsus were then determined using alcian blue and alizarin red double staining (Fig. 6a). Also, we observed alizarin red positive staining in all of the radius, and femur. The length of the radius stained with alizarin red was measured between control and HSYA treatments and statistical analyzed (control: $70.78 \pm 25.89 \mathrm{~mm}$; HSYA: 105.70 $\pm 14.09 \mathrm{~mm} ; \mathrm{n}=12$ for each group). For the femur the extent of staining was control: $64.53 \pm 22.15 \mathrm{~mm}$; HSYA: $90.63 \pm 27.53 \mathrm{~mm}$ (Fig. 6b). These results implied that HSYA exposure during embryogenesis significantly accelerates bone mineralization.

\section{HSYA protected against OVX-induced bone loss}

Having established that HSYA has an effect on enhancing osteoblast formation, we then investigated the potential of HSYA as a preventive agent to prevent OVX-induced osteoporosis in vivo. Rats were OVX- or sham-operated and then injected with HSYA $(2.5 \mathrm{mg} / \mathrm{kg}$ or $10 \mathrm{mg} / \mathrm{kg})$ every $2-3$ days, or PBS for 12 weeks post-surgery. Micro-CT analysis showed that the HSYA prevented the extensive bone mass loss in the OVX rat model. Quantitative analysis confirmed that bone parameters, including BV/TV, Tb. N, Tb. Th, BMD and Tb. Sp, were measured in the HSYA treatment group (Fig. 7a-b).

The H\&E staining results also confirmed the improved trabecular structure in different groups after HSYA intervention. Immunohistochemistry showed that HSYA significantly stimulated the expression of Bglap in the bone (Fig. 8).

\section{Discussion}

Bone is a kind of tissue remodeled constantly. Under normal circumstances, there is a balance between bone formation and bone resorption, which is essential for maintaining bone quality and compressive stress characteristic. This balance can be achieved by regulating the activity of osteoblasts and osteoclasts, which are responsible for bone formation and absorption, respectively. However, under certain pathological conditions, an imbalance between bone resorption and bone formation may occur, leading to abnormal bone remodeling and the development of bone diseases such as osteoporosis. Clinically, this manifests as age-related osteoporosis associated with bone loss and increased fat accumulation [24].

Mesenchymal stem cells which are capable to multi-directionally differentiate can be easily cultured and expanded. However, the ability of MSCs to differentiate into functional osteoblasts is still limited in vivo [25]. The main reason is the adipogenic tendency of the precursor cell can suppress the tendency of osteogenic differentiation then breaks this bidirectional balance to cause osteoporosis [26]. Once osteoblast differentiation is abnormal, it will have an important impact on bone metabolism balance. On 
the other hand, osteoclasts responsible for bone resorption evolve from the lineage of monocytes differentiated by hematopoietic stem cells, and their differentiation process is also regulated by a variety of factors. Multiple studies have shown that the expression of PPARY, C/EBPa and C/EBP $\beta$ in hematopoietic cells are essential for activating osteoclast differentiation and maturation and can directly affect the osteoclastogenesis process of hematopoietic stem cells [27-29]. This indicates that adipogenic differentiation of MSCs can directly promote osteoclastogenesis and bone resorption by breaking the coupling balance between osteoblasts and osteoclasts, which in turn leads to the occurrence of osteoporosis. Therefore, promoting the osteogenic differentiation of MSCs and inhibiting their adipogenic differentiation to correct the imbalance of bone metabolism is one of the directions for the treatment of osteoporosis.

In this study, we demonstrated for the first time that HSYA enhance osteogenic effect of hBMSCs through KDM7A regulating histone demethylation in $\beta$-catenin in vitro. In vivo, HSYA can promote skeletal development and prevent the development of OVX-induced osteoporosis rats' model in vivo.

In vitro, the results from current study showed that HSYA did not affect the viability of hBMSCs on a wide range of concentrations, indicating that HSYA had no toxicity to hBMSCs. In order to evaluate the effect of HSYA on osteoblastic differentiation of hBMSCs, as previously [30], we first investigated the role of HSYA on ALP activity, an early marker of osteoblastic differentiation. ALP hydrolyzes pyrophosphate to phosphate, which reacts with calcium to form hydroxyapatite to promote mineralization, suggesting that ALP has a huge positive impact on bone formation [31]. Our result indicated that HSYA significantly increased the activity of ALP in hBMSCs. As expected, HSYA also enhanced calcium nodule formation, a functional marker of mineralization [32]. These results showed that HSYA could promote the osteogenic ability of hBMSCs.

Next, we checked the changes of Runx2 and $\beta$-catenin in HSYA treated hBMSCs. The runt family transcription factor Runx 2 is a key transcriptional regulator and plays a key role in osteoblast differentiation [33]. Osteoblast differentiation normally occurs in Run $2^{+} \mathrm{OSX} \mathrm{X}^{-}$mesenchymal cells, while in Runx2-deleted embryos, osteoblast differentiation is inhibited, indicating that Runx 2 is a key transcription factor for osteogenesis [34]. Furthermore, Wnt/ $\beta$-catenin signaling directly enhances Runx2 through both canonical and non-canonical pathways, leading to bone formation ultimately [35]. Western blotting showed HSYA increased the Runx2 and $\beta$-catenin, suggesting that HSYA might promote osteogenic differentiation of hBMSCs through mediating $\beta$-catenin. To verify that, we found luciferase activity of Wnt signaling reporter TOPflash and nucleus translocation of $\beta$-catenin were activated significantly by $10 \mu \mathrm{M}$ HSYA, which meant $\beta$-catenin might be indicative of a key role of HSYA in promoting osteogenesis in hBMSCs.

Based on the above results, we concluded that $10 \mu \mathrm{M}$ HSYA is the most suitable dose to promote the osteogenesis of hBMSCs. Therefore, RNA-sequencing analysis was used for the exploration of the mechanisms underlying the osteogenesis effects of HSYA. On the basis of previous report [12], we found HXTL capsule which contains Safflower mainly could promote osteogenesis of hBMSCs to ameliorate 
osteonecrosis of femoral head through histone demethylation in IncRNA-Miat. Therefore, we hypothesized the bone formation of HSYA is likely to be dependent on histone demethylation, and chose six KDMs orthologous genes with differential expression, coding their namesake proteins with the histone demethylase signature domain Jumonji C (JmjC), for further verification from the results of RNAsequencing analysis. We found that KDM7A was the potential one to be targeted as its expression was significantly up-regulated in HSYA groups.

Histone lysine (K)-specific demethylase 7A (KDM7A) has been proved to remove di-methylation marks of histone H3K9 and H3K27 [36]. Previous research reported that KDM7A can suppress tumours through blocking tumours growth and angiogenesis and regulate neural differentiation and fibroblast growth factor-4 (FGF-4) expression. To clarify the precise role of KDM7A in osteogenesis of hBMSCs, we tested the effects of siRNA-KDM7A, the related markers including $\beta$-catenin were down-regulated in the absence of KDM7A through various experiments. Furthermore, HSYA can reverse this effect to some extent.

The effects of H3K27me2/H3K9me2 on regulating osteogenic differentiation are investigated in recent years, and $\mathrm{H} 3 \mathrm{~K} 9 \mathrm{me} 2$ is supposed to be concluded that it has the impact on differentiation of hBMSCs $[37,38]$. The cell immunofluorescence showed that HSYA could inhibit H3K27me2/H3K9me2 expression, which are the targets of KMD7A to be removed on the promoters of related genes [39]. And the CHIP assay showed that the binding ratio between $\mathrm{H} 3 \mathrm{~K} 27 \mathrm{me} 2$ and promoter of $\beta$-catenin was partly blocked by HSYA interference, suggesting the osteogenesis effect of HSYA in hBMSCs is likely to be associated with histone dimethylation in promoter of $\beta$-catenin.

A recent study reports that KDM7A is a molecular regulator for adipogenic and osteogenic differentiation in stromal ST2 cell and mBMSCs line. The investigators suggest that KDM7A can inhibit osteogenesis through epigenetic control of C/EBPa and canonical Wnt signaling [40]. However, our results are in stark contrast because we provide evidence from knockdown of KDM7A that it is a positive factor on osteogenesis in vitro in hBMSCs. Under the condition that HSYA can enhance osteogenetic differentiation of hBMSCs, KDM7A was verified to be one potential mediator and contributor. Moreover, we showed that the H3K9m2 and H3K27m2 can be influenced by HSYA. There exist two main reasons can explain the differences: the KDM7A expression has not reached the peak until 8 days either in primary BMSCsosteogenetic or ST2- osteogenetic, comparing with the peak expression of KDM7A in ST2-Adipogenic in 3 day. However, in subsequent experiments of that reports, the related genes and proteins markers were test within 3 days, which forms the contrast to ours (7 day). So, the timing of termination of culture and collection of cell samples may be one reason. In addition, the cell lines and vitro differentiation conditions are likely to be the causes of this opposite results as these two reports [41, 42].

Based on the vitro results, we further evaluated overall the biological function in accelerated mineralization of the bone and osteogenesis of HSYA through Chick Embryos. This was clearly demonstrated by the extent of calcification of the spine, radius, metatarsus and femur, which proved the promotion effect of HSYA on bone development, and in accordance with osteogenic ability of HSYA in hBMSCs basing on the theory of Cartilaginous Internalized Bone. Finally, we established an OVX rat 
model to further investigate whether HSYA has potential therapeutic effect in vivo. We can conclude that HSYA exhibit a remarkable protective effect on OVX-induced bone loss in a rat model as confirmed by micro-CT and histological and immunohistochemical analyses, and the relevant results were consistent with the in vitro study.

\section{Conclusion}

Taken together, our study has demonstrated for the first time that HSYA can accelerate osteogenesis of hBMSCs via histone demethylation, and $\beta$-catenin is likely to be one target which further attenuates downstream osteogenesis gene expressions (Fig. 9). More importantly, HSYA is capable to promote bone development and suppress ovariectomized osteoporosis. In conclusion, these findings could pave the way to the potential development of HSYA-targeted therapeutic treatments for skeletal diseases such as osteoporosis.

\section{Abbreviations}

MSCs

Mesenchymal stem cells; HXTL capsule:Huo Xue Tong Luo capsule; HSYA:Hydroxysafflor yellow A; hBMSCs:human bone marrow-derived mesenchymal stem cells; OVX:ovariectomy; Micro-CT:microcomputed tomography; TBST:TBS containing $0.1 \%$ Tween 20; OIM:Osteogenic induction medium

\section{Declarations}

\section{Acknowledgements}

Not applicable

\section{Author' contributions}

LLX, HBW, andGL conceived, designed, supervised, and commented on all the drafts of this paper. PW, TLZ, YL, MZ and CZ conducted the overall experiments; participated in the data collection, analysis, and molecular investigations, and helped in the drafts. JFZ and GL provided the TOPflash and Renilla reporter plasmid pRL-CMV. All authors read and approved the final version of the manuscript.

\section{Funding}

The work was supported by grants from National Natural Science Foundation of China (NSFC No. 81774339, 81871778), Guangdong Provincial Science and Technology Project (No. 2017A050506046). The funding institutions had not any role in study design, data collection, data analysis, interpretation nor writing of the report in this study.

\section{Availability of data and materials}


The datasets used and/or analyzed during the current study are availablefrom the corresponding author on reasonable request

\section{Ethics approval and consent to participate}

The mouse model testing involved in our study followed the BaselDeclaration outlines fundamental principles and was approved by the Institutional Animal Ethics Committee of the First Affiliated Hospital, Guangzhou University of Chinese Medicine (TCMF1-2018011).

\section{Consent for publication}

Not applicable.

\section{Competing interests}

The authors declare that they have no competing interests

\section{Author details}

${ }^{1}$ Guangzhou University of Chinese Medicine, Guangzhou, China. ${ }^{2}$ Department of Joint Orthopaedics, the First Affiliated Hospital, Guangzhou University of Chinese Medicine, Guangzhou, Guangdong 510405, China. ${ }^{3}$ Laboratory of Orthopaedics\& Traumatology, Lingnan Medical Research Center, Guangzhou University of Chinese Medicine, Guangzhou, China. ${ }^{4}$ Department of Orthopaedics and Traumatology, Faculty of Medicine, Prince of Wales Hospital, The Chinese University of Hong Kong, Shatin, Hong Kong, Special Administrative Region of China

\section{References}

1. Cohen MM. Jr. The new bone biology: pathologic, molecular, and clinical correlates. American journal of medical genetics Part A. 2006;140(23):2646-706.

2. Cheng X, Yan Y, Chen JL, Ma ZL, Yang RH, Wang G, et al. Dexamethasone Exposure Accelerates Endochondral Ossification of Chick Embryos Via Angiogenesis. Toxicol Sci. 2016;149(1):167-77.

3. Yousefi AM, James PF, Akbarzadeh R, Subramanian A, Flavin C, Oudadesse H. Prospect of Stem Cells in Bone Tissue Engineering: A Review. Stem Cells Int. 2016; 2016: 6180487.

4. Takada I, Kouzmenko AP, Kato S. Molecular switching of osteoblastogenesis versus adipogenesis: implications for targeted therapies. Expert Opin Ther Targets. 2009;13(5):593-603.

5. Huang W, Yang S, Shao J, Li YP. Signaling and transcriptional regulation in osteoblast commitment and differentiation. Front Biosci. 2007;12:3068-92.

6. Nusse R, Clevers H. Wnt/beta-Catenin Signaling, Disease, and Emerging Therapeutic Modalities. Cell. 2017;169(6):985-99.

7. Day TF, Guo X, Garrett-Beal L, Yang Y. Wnt/beta-catenin signaling in mesenchymal progenitors controls osteoblast and chondrocyte differentiation during vertebrate skeletogenesis. Dev Cell. 
2005;8(5):739-50.

8. Chen Y, Whetstone HC, Lin AC, Nadesan P, Wei Q, Poon R, et al. Beta-catenin signaling plays a disparate role in different phases of fracture repair: implications for therapy to improve bone healing. PLoS Med. 2007;4(7):e249.

9. Holmen SL, Zylstra CR, Mukherjee A, Sigler RE, Faugere MC, Bouxsein ML, et al. Essential role of betacatenin in postnatal bone acquisition. J Biol Chem. 2005;280(22):21162-8.

10. Chen R, Lee WY, Zhang XH, Zhang JT, Lin S, Xu LL, et al. Epigenetic Modification of the CCL5/CCR1/ERK Axis Enhances Glioma Targeting in Dedifferentiation-Reprogrammed BMSCs. Stem Cell Reports. 2017;8(3):743-57.

11. Wang P, Cao Y, Zhan D, Wang D, Wang B, Liu Y, et al. Influence of DNA methylation on the expression of OPG/RANKL in primary osteoporosis. Int J Med Sci. 2018;15(13):1480-5.

12. Fang B, Li Y, Chen C, Wei Q, Zheng J, Liu Y, et al. Huo Xue Tong Luo capsule ameliorates osteonecrosis of femoral head through inhibiting IncRNA-Miat. J Ethnopharmacol. 2019;238:111862.

13. Ko JY, Chuang PC, Chen MW, Ke HC, Wu SL, Chang YH, et al. MicroRNA-29a ameliorates glucocorticoid-induced suppression of osteoblast differentiation by regulating beta-catenin acetylation. Bone. 2013;57(2):468-75.

14. Jiang C, He C, Wu Z, Li F, Xiao J. Histone methyltransferase SETD2 regulates osteosarcoma cell growth and chemosensitivity by suppressing Wnt/ $\beta$-catenin signaling. Biochem Biophys Res Commun. 2018;502(3):382-8.

15. Sen B, Paradise CR, Xie Z, Sankaran J, Uzer G, Styner M, et al. $\beta$-Catenin Preserves the Stem State of Murine Bone Marrow Stromal Cells Through Activation of EZH2. Journal of bone and mineral research: the official journal of the American Society for Bone and Mineral Research. 2020.

16. Liang WC, Fu WM, Wang YB, Sun YX, Xu LL, Wong CW, et al. H19 activates Wnt signaling and promotes osteoblast differentiation by functioning as a competing endogenous RNA. Scientific reports. 2016;6:20121.

17. Kim JH, He MT, Kim MJ, Yang CY, Shin YS, Yokozawa T, et al. Safflower (Carthamus tinctorius L.) seed attenuates memory impairment induced by scopolamine in mice via regulation of cholinergic dysfunction and oxidative stress. Food Funct. 2019;10(6):3650-9.

18. Ao H, Feng W, Peng C. Hydroxysafflor Yellow A: A Promising Therapeutic Agent for a Broad Spectrum of Diseases. Evid Based Complement Alternat Med. 2018; 2018: 8259280.

19. Ma L, Liu L, Ma Y, Xie H, Yu X, Wang X, et al. The Role of E-Cadherin/beta-Catenin in Hydroxysafflor Yellow A Inhibiting Adhesion, Invasion, Migration and Lung Metastasis of Hepatoma Cells. Biol Pharm Bull. 2017;40(10):1706-15.

20. Lv MY, Shi CJ, Pan FF, Shao J, Feng L, Chen G, et al. Urolithin B suppresses tumor growth in hepatocellular carcinoma through inducing the inactivation of Wnt/ $\beta$-catenin signaling. Journal of cellular biochemistry. 2019;120(10):17273-82.

21. Fang B, Wang D, Zheng J, Wei Q, Zhan D, Liu Y, et al. Involvement of tumor necrosis factor alpha in steroid-associated osteonecrosis of the femoral head: friend or foe? Stem Cell Res Ther. 
2019;10(1):5.

22. Hamburger V, Hamilton HL. A series of normal stages in the development of the chick embryo. 1951. Dev Dyn. 1992;195(4):231-72.

23. Schmitz N, Laverty S, Kraus VB, Aigner T. Basic methods in histopathology of joint tissues. Osteoarthritis Cartilage. 2010;18(Suppl 3):113-6.

24. Li Y, He X, Li Y, He J, Anderstam B, Andersson G, et al. Nicotinamide phosphoribosyltransferase (Nampt) affects the lineage fate determination of mesenchymal stem cells: a possible cause for reduced osteogenesis and increased adipogenesis in older individuals. Journal of bone mineral research: the official journal of the American Society for Bone Mineral Research. 2011;26(11):265664.

25. Xu L, Huang S, Hou Y, Liu Y, Ni M, Meng F, et al. Sox11-modified mesenchymal stem cells (MSCs) accelerate bone fracture healing: Sox11 regulates differentiation and migration of MSCs. FASEB J. 2015;29(4):1143-52.

26. Li CJ, Cheng P, Liang MK, Chen YS, Lu Q, Wang JY, et al. MicroRNA-188 regulates age-related switch between osteoblast and adipocyte differentiation. J Clin Investig. 2015;125(4):1509-22.

27. Muruganandan S, Govindarajan R, Sinal CJ. Bone Marrow Adipose Tissue and Skeletal Health. Curr Osteoporos Rep. 2018;16(4):434-42.

28. Jules J, Chen W, Feng X, Li YP. CCAAT/Enhancer-binding Protein a (C/EBPa) Is Important for Osteoclast Differentiation and Activity. J Biol Chem. 2016;291(31):16390-403.

29. Chen W, Zhu G, Tang J, Zhou HD, Li YP. C/ebpa controls osteoclast terminal differentiation, activation, function, and postnatal bone homeostasis through direct regulation of Nfatc1. J Pathol. 2018;244(3):271-82.

30. Xu D, Xu L, Zhou C, Lee WY, Wu T, Cui L, et al. Salvianolic acid B promotes osteogenesis of human mesenchymal stem cells through activating ERK signaling pathway. Int J Biochem Cell Biol. 2014;51:1-9.

31. Orimo $\mathrm{H}$. The mechanism of mineralization and the role of alkaline phosphatase in health and disease. Journal of Nippon Medical School = Nippon Ika Daigaku zasshi. 2010;77(1):4-12.

32. Gordon JA, Tye CE, Sampaio AV, Underhill TM, Hunter GK, Goldberg HA. Bone sialoprotein expression enhances osteoblast differentiation and matrix mineralization in vitro. Bone. 2007;41(3):462-73.

33. Yin W, Liu S, Dong M, Liu Q, Shi C, Bai H, et al. A New NLRP3 Inflammasome Inhibitor, Dioscin, Promotes Osteogenesis. Small. 2020;16(1):e1905977.

34. Molagoda IMN, Karunarathne W, Choi YH, Park EK, Jeon YJ, Lee BJ, et al. Fermented Oyster Extract Promotes Osteoblast Differentiation by Activating the Wnt/beta-Catenin Signaling Pathway, Leading to Bone Formation. Biomolecules. 2019; 9(11).

35. Lerner UH, Ohlsson C. The WNT system: background and its role in bone. J Intern Med. 2015;277(6):630-49. 
36. Pan MR, Hsu MC, Chen LT, Hung WC. G9a orchestrates PCL3 and KDM7A to promote histone H3K27 methylation. Scientific reports. 2015;5:18709.

37. Tsukada Y, Ishitani T, Nakayama KI. KDM7 is a dual demethylase for histone H3 Lys 9 and Lys 27 and functions in brain development. Genes Dev. 2010;24(5):432-7.

38. Wang L, Xu S, Lee JE, Baldridge A, Grullon S, Peng W, et al. Histone H3K9 methyltransferase G9a represses PPARgamma expression and adipogenesis. EMBO J. 2013;32(1):45-59.

39. Jang MK, Kim JH, Jung MH. Histone H3K9 Demethylase JMJD2B Activates Adipogenesis by Regulating H3K9 Methylation on PPARgamma and C/EBPalpha during Adipogenesis. PloS one. 2017;12(1):e0168185.

40. Yang X, Wang G, Wang Y, Zhou J, Yuan H, Li X, et al. Histone demethylase KDM7A reciprocally regulates adipogenic and osteogenic differentiation via regulation of $\mathrm{C} / \mathrm{EBPa}$ and canonical Wnt signalling. J Cell Mol Med. 2019;23(3):2149-62.

41. Dey BK, Pfeifer K, Dutta A. The H19 long noncoding RNA gives rise to microRNAs miR-675-3p and miR-675-5p to promote skeletal muscle differentiation and regeneration. Genes Dev. 2014;28(5):491-501.

42. Kallen AN, Zhou XB, Xu J, Qiao C, Ma J, Yan L, et al. The imprinted H19 IncRNA antagonizes let-7 microRNAs. Mol Cell. 2013;52(1):101-12.

\section{Figures}

A

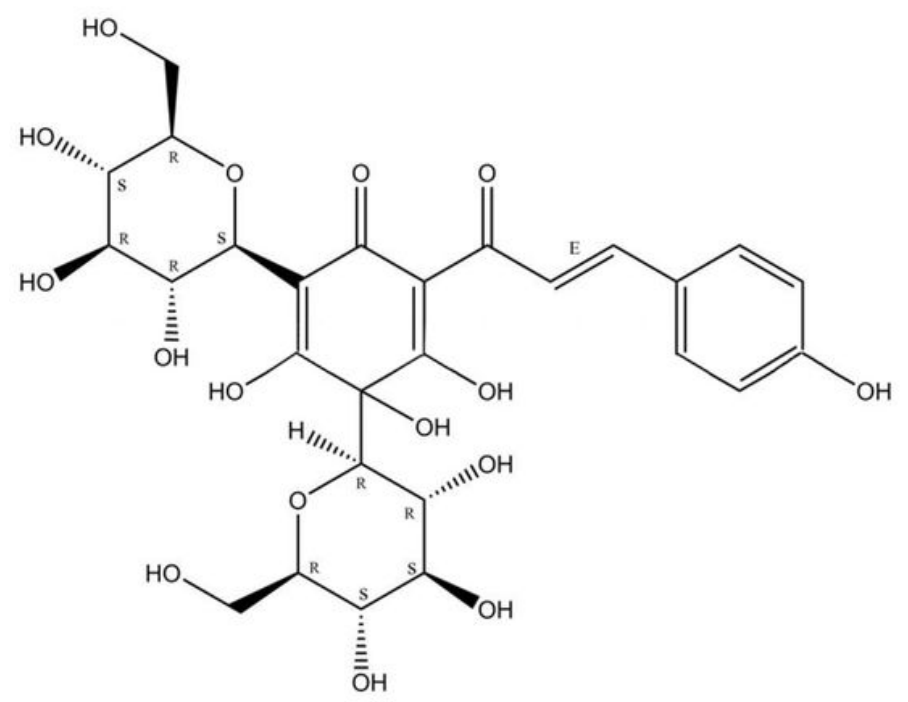

B

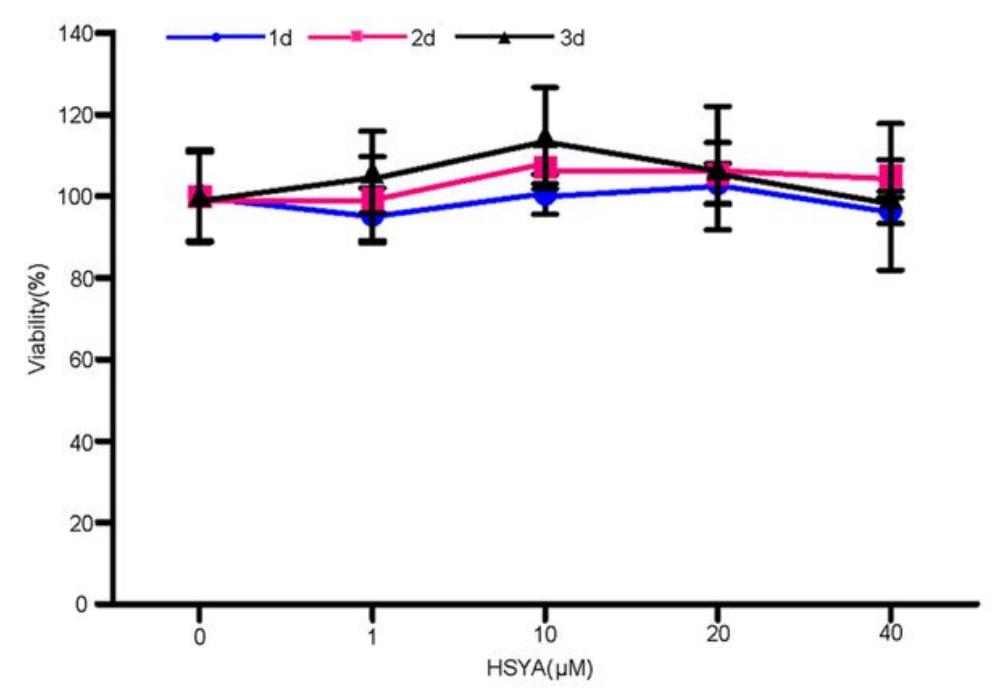

\section{Figure 1}

Viability of hMSCs treated by HSYA at different concentrations. aThe basic chemical structure of HSYA. bThe cells were incubated with HSYA $(1-40 \mu \mathrm{M})$ for 1,2 and 3 days, and then CCK-8 assay was performed to test the cell viability. 
A

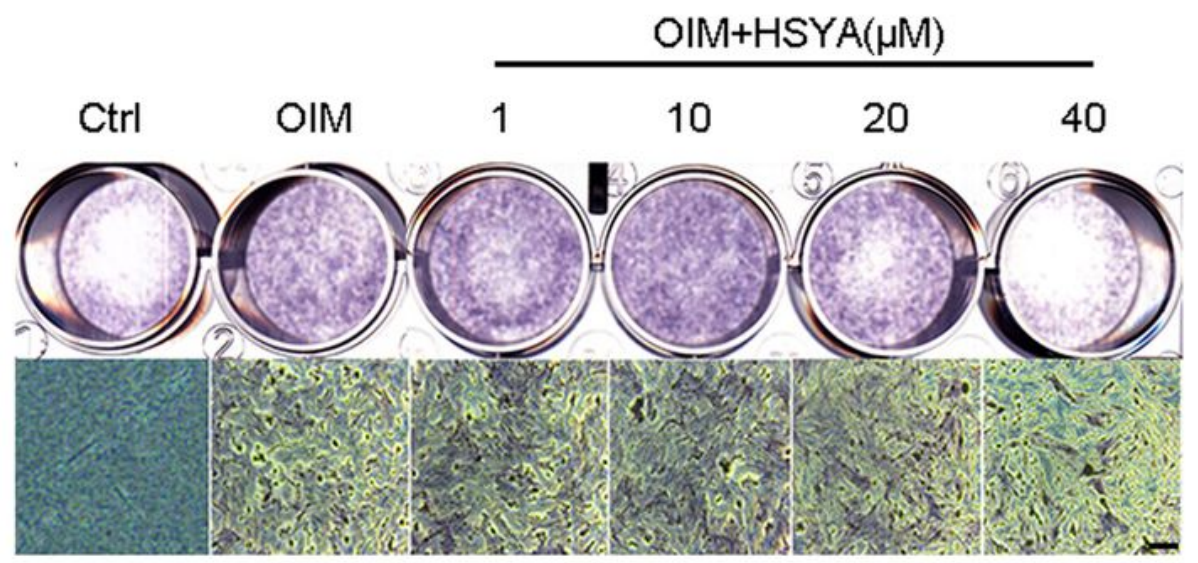

B

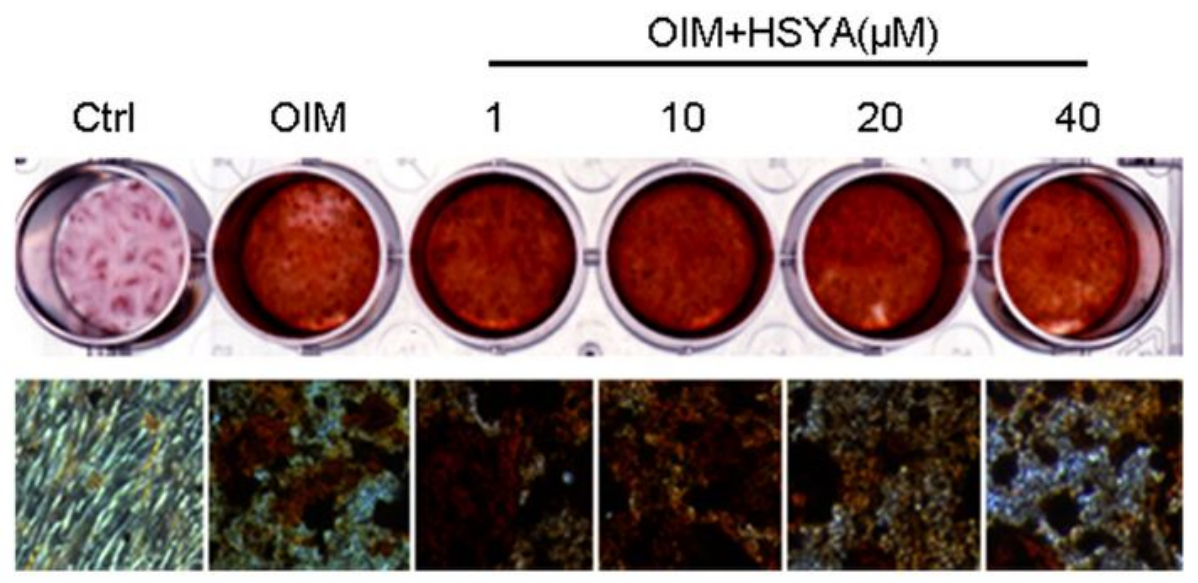

C

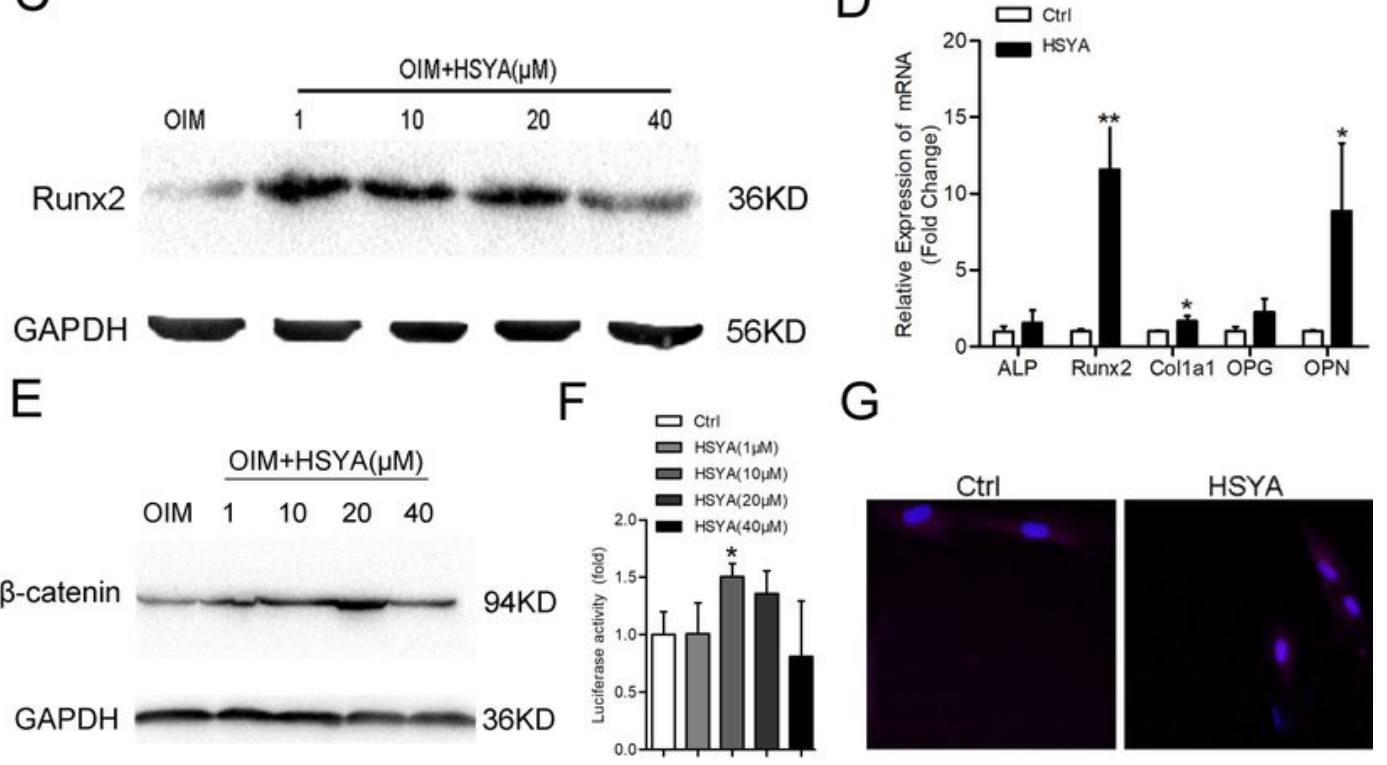

Figure 2

HSYA promotes osteogenesis of hBMSCs through targeting $\beta$-catenin.aALP in hBMSCs was stained with BCIP/NBT kit after the cells were incubated with different concentrations of HSYA in OIM for 7 days. bhBMSCs were treated with HSYA $(1-40 \mu \mathrm{M})$ in OIM for 14 days, and then the mineralized nodules were stained by Alizarin Red. Scale bars $=200 \mu \mathrm{m}$. chBMSCs were treated with $\operatorname{HSYA}(1,10,20,40) \mu \mathrm{M}$ in OIM for 7 days, the protein levels of Runx 2 was detected by western blotting with the indicated antibodies. 
GAPDH was used as loading control. dhBMSCs were treated with HSYA $(10 \mu \mathrm{M})$ in OIM for 7 days, then gene expressions were detected by real time PCR. $\beta$-actin was used as an internal control. ${ }^{*} P<0.05$, ${ }^{*} \mathrm{P}<0.01$, compared with Ctrl. ehBMSCs were treated with $\operatorname{HSYA}(1,10,20,40) \mu \mathrm{M}$ in OIM for 7 days, the protein level of $\beta$-catenin was detected by western blotting with the indicated antibodies. GAPDH was used as loading control. fThe TOPflash luciferase activity was measured in hBMSCs after treatment of HSYA at different concentrations. ${ }^{*} \mathrm{P}<0.05$, compared with Ctrl. ghBMSCs were treated with HSYA $(10 \mu M)$ for $48 \mathrm{~h}$, and then $\beta$-catenin was detected by immunofluorescence staining.
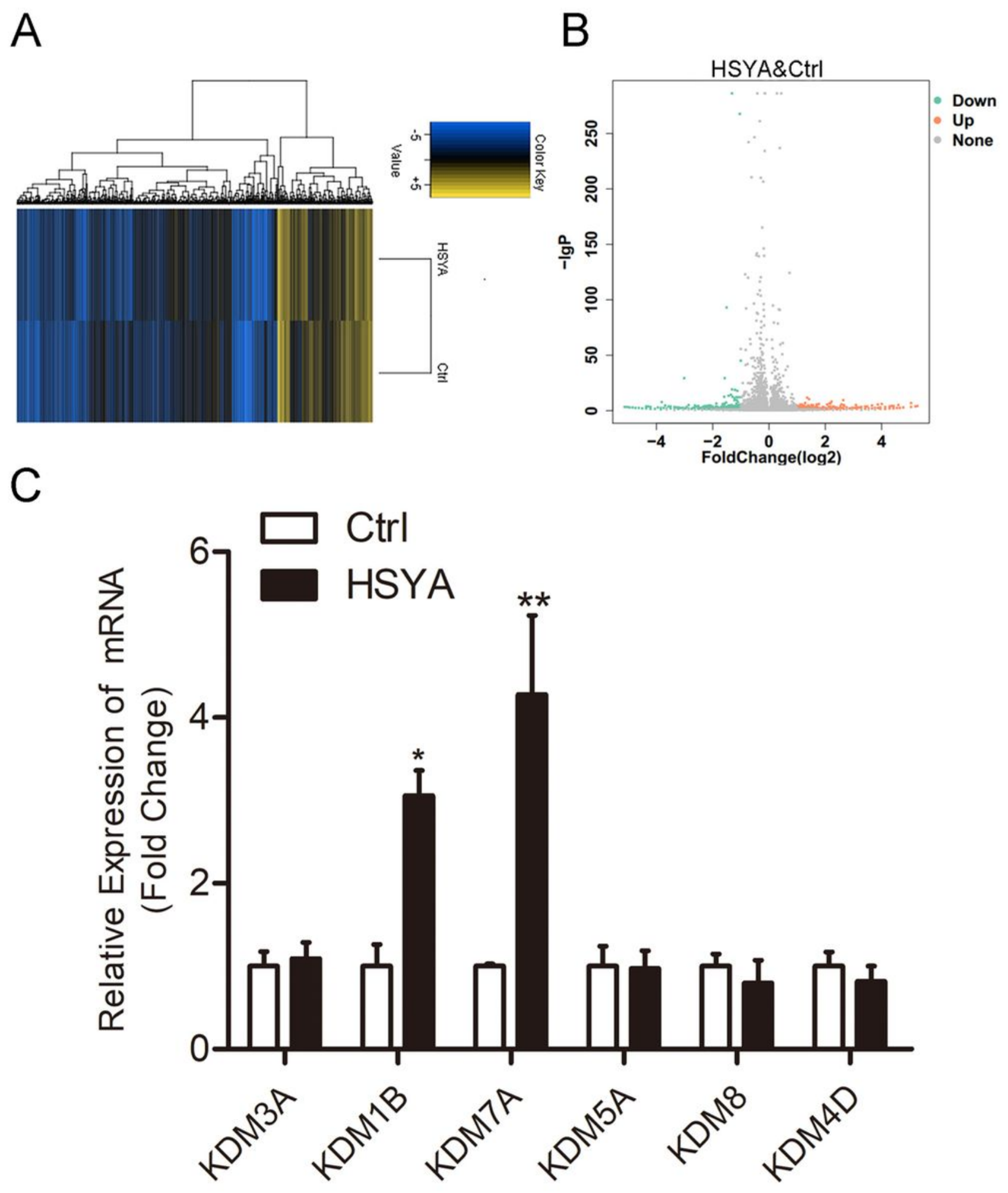


\section{Figure 3}

HSYA regulates the KDMs genes.aHeatmap depicting expression levels of genes between PBS- and HSYA-treated hBMSCs.bVolcano map of the differentially expressed genes in PBS- and HSYA-treated hBMSCs.cEvaluate the expression levels of human KDM3A/1B/7A/5A/8/4D in PBS- and HSYA-treated hBMSCs by quantitative RT-PCR. $\beta$-actin was used as an internal control. ${ }^{*} P<0.05$, ${ }^{*} P<0.01$, compared with Ctrl.
A
B
C
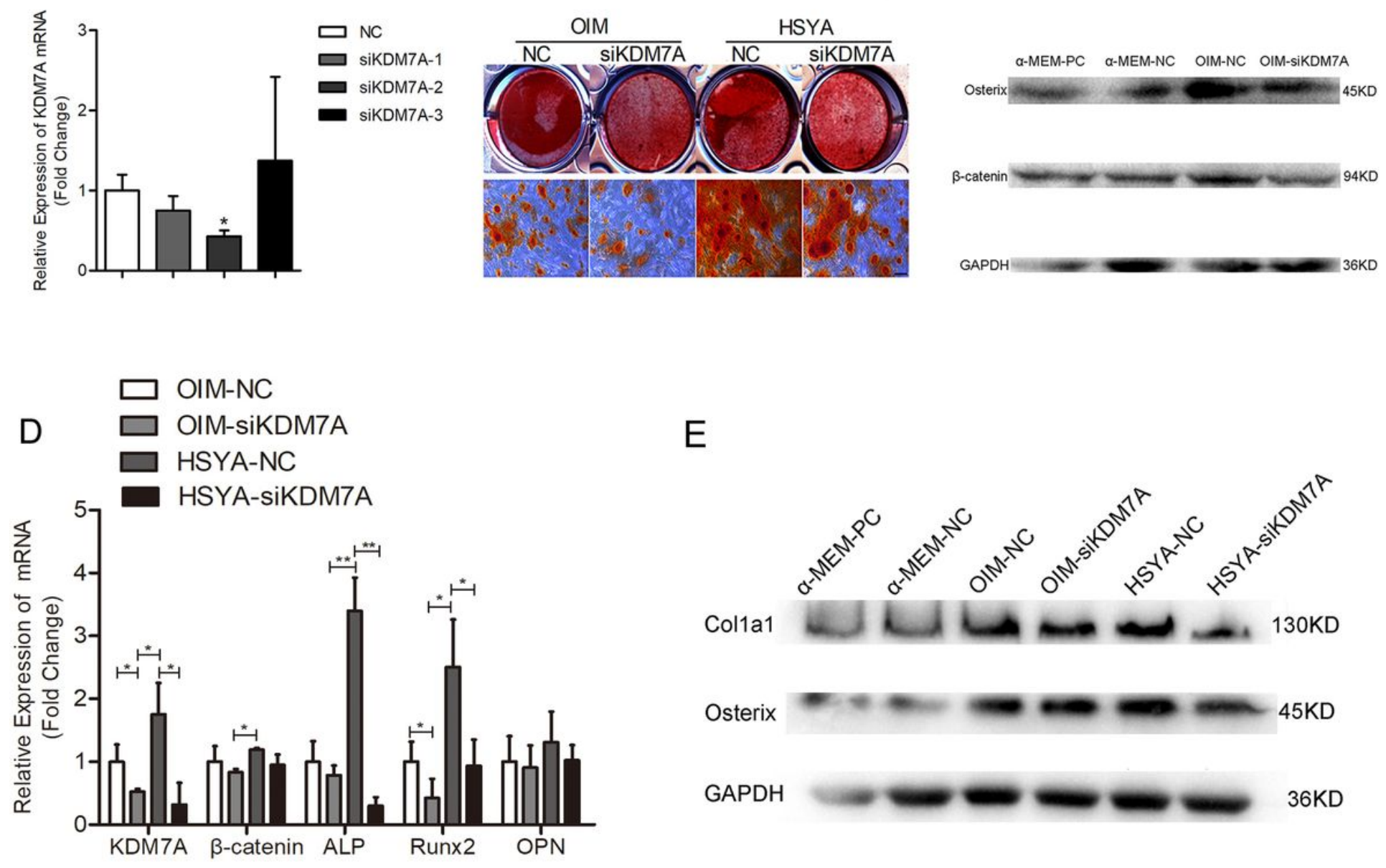

$\mathrm{E}$

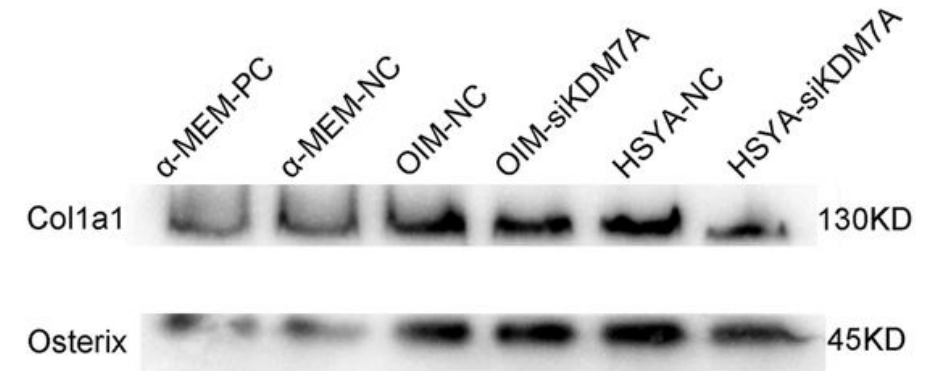

GAPDH

36KD

\section{Figure 4}

KDM7A plays an epigenetic role in mechanism of osteogenesis effect of HSYA.aThe siRNA targeting KDM7A was transfected into hBMSCs as mentioned in Methods and Materials. ${ }^{*} \mathrm{P}<0.05$, compared with NC group. bhBMSCs were transduced with siRNA-KDM7A or NC. The mineralized nodules of hBMSCs were stained by Alizarin Red S after 14 days of osteogenic induction. Scale bars $=200 \mu \mathrm{m}$. cThe total proteins were extracted from hBMSCs transduced with siRNA-KDM7A or NC, and separated by $10 \%$ SDSPAGE and analyzed by western blotting with specific antibodies. PC, positive control. dhBMSCs were transduced with siRNA-KDM7A or NC. Then the cells were incubated with or not 10 $\mu \mathrm{M}$ HSYA in OIM for 7 days. Then the expression levels of KDM7A, ALP, Runx2, OPN and $\beta$-catenin were detected by real time PCR. $\beta$-actin was used as an internal control. ${ }^{*} P<0.05,{ }^{*} P<0.01$, compared with OIM-siKDM7A, HSYAsiKDM7A groups accordingly. ehBMSCs transduced with siRNA-KDM7A or NC. Then the cells were 
incubated with or not $10 \mu \mathrm{M}$ HSYA in OIM for 7 days. The total proteins were extracted from hBMSCs to be analyzed by western blotting with specific antibodies.

A
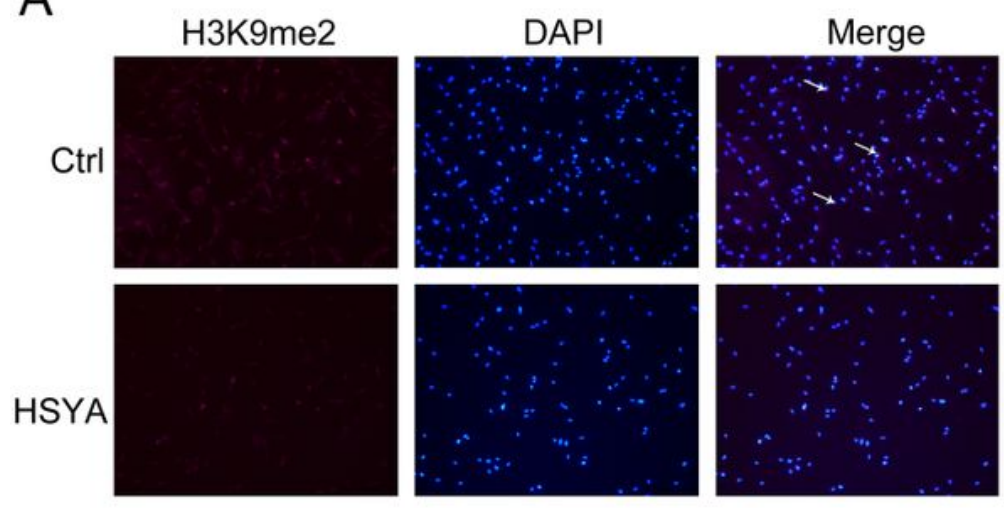

B
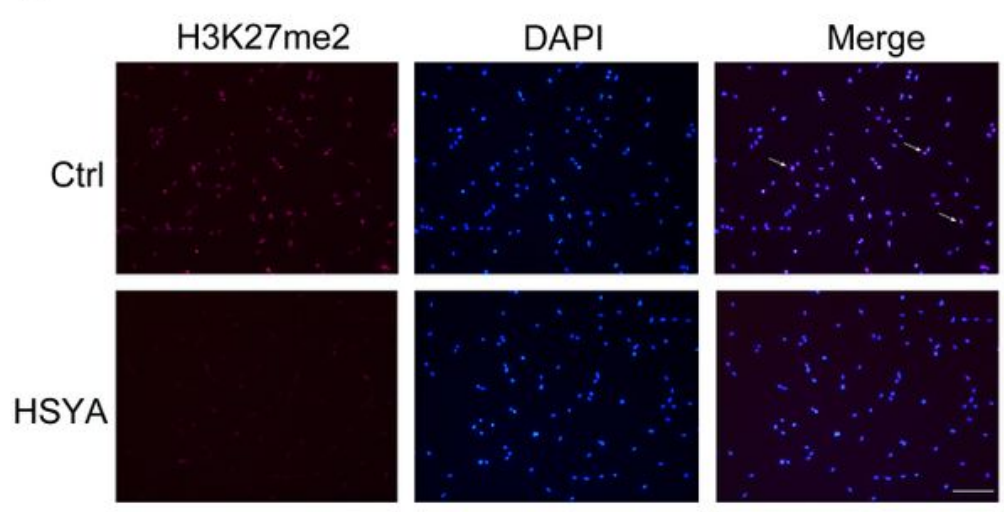

C

Human $\beta$-catenin promoter
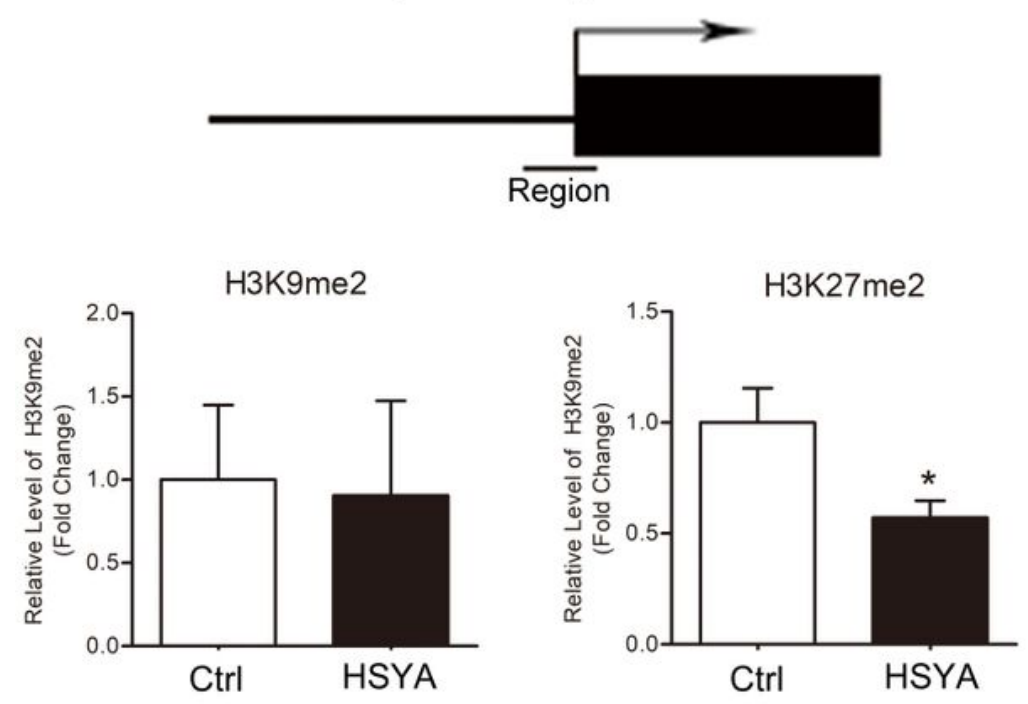

Figure 5

HSYA regulates $\beta$-catenin expression in hMSCs by histone demethylation. a-bhBMSCs were treated with HSYA $(10 \mu \mathrm{M})$ for $48 \mathrm{~h}$, and then H3K9me2\&H3K27me2 were detected by immunofluorescence staining. Scale bars $=200 \mu \mathrm{m} . c$ Decreased occupancy of H3K9me2 and significantly increased occupancy of 
H3K27me2 on human $\beta$-catenin promoter in hMSCs treated with HSYA. The histone modifications of human $\beta$-catenin promoter were analyzed by the ChIP-PCR assay. ChIP was done using anti-H3K9me2 and anti-H3K27me2 monoclonal antibodies, and PCR was performed with specific primers. Normal rabbit IgG was used as loading control. ${ }^{*} \mathrm{P}<0.05$, compared with $\mathrm{Ctrl}$ group.

A

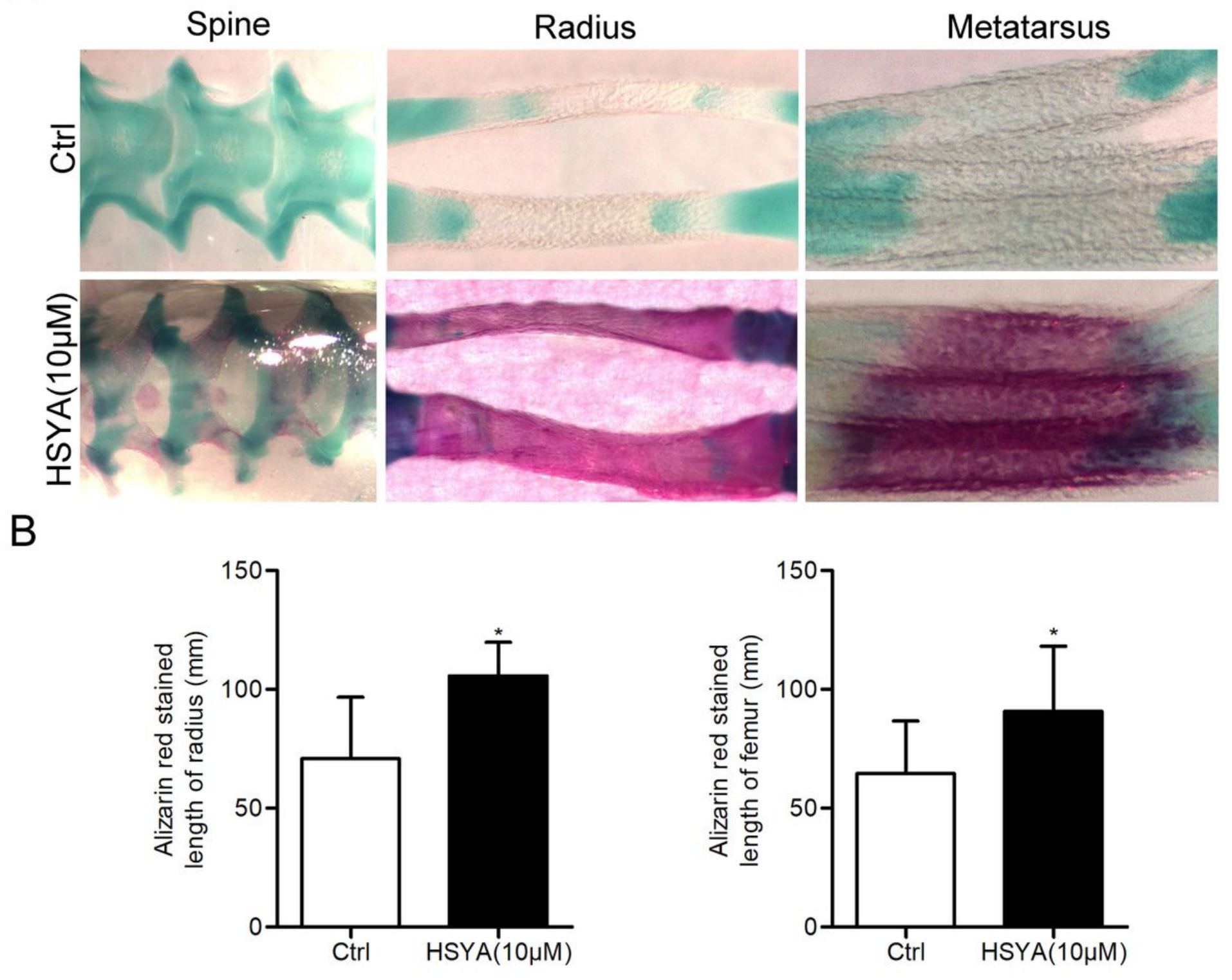

Figure 6

HSYA exposure during gastrulation accelerates long bone mineralization.aRepresentative appearance of chick embryos' spine, radius, metatarsus treated with DMSO (Ctrl) or 10 $\mu \mathrm{M}$ HSYA. b Bar charts comparing the length of alizarin red radius, and femur between control and HSYA-treated embryos. ${ }^{*}<<0.05$, compared with Ctrl. 
A

Sham
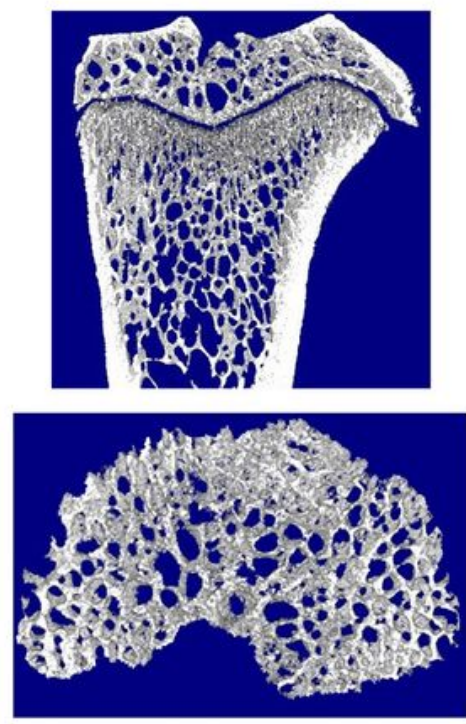

PBS
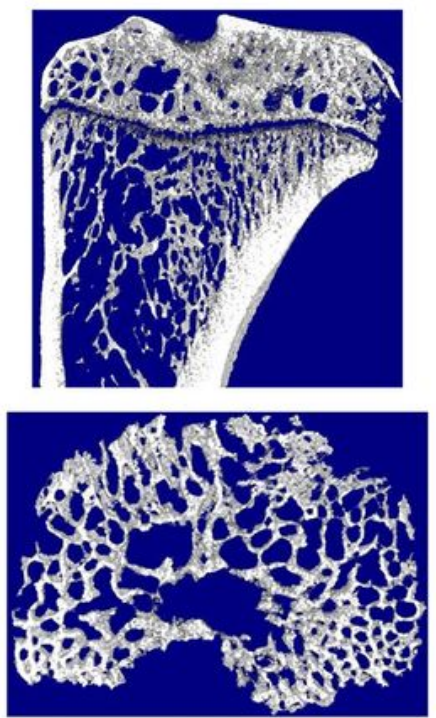

$\mathrm{HSYA}(2.5 \mathrm{mg} / \mathrm{kg})$
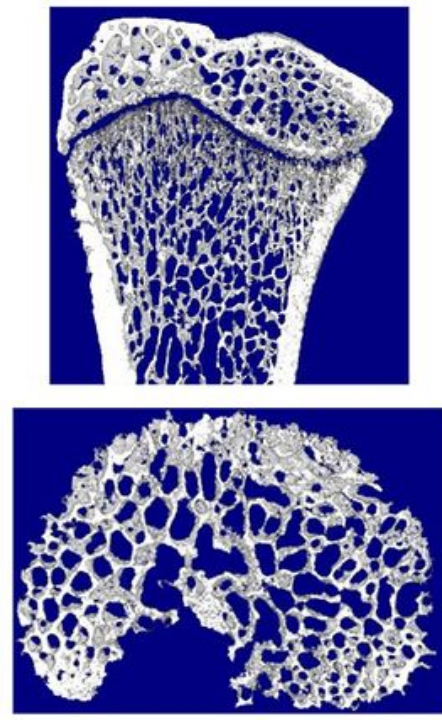

$\operatorname{HSYA}(10 \mathrm{mg} / \mathrm{kg})$
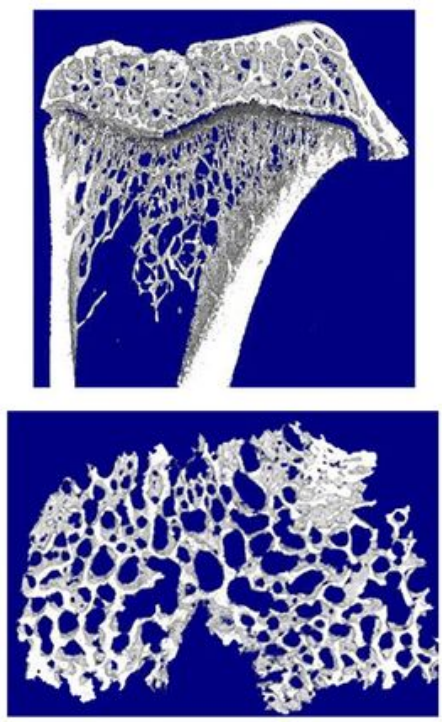

B
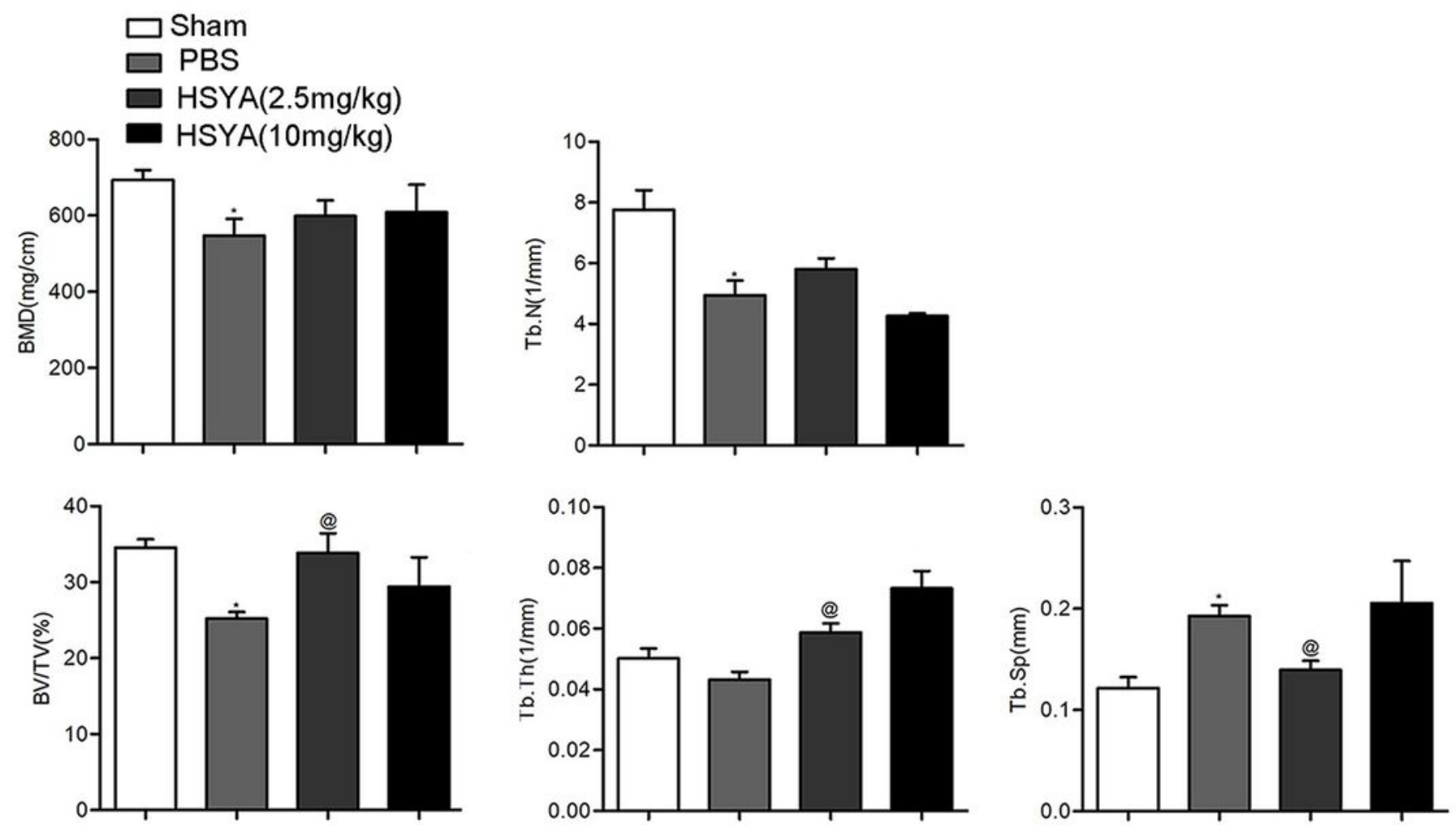

Figure 7

HSYA prevents OVX-induced bone mass loss in vivo. aRepresentative Micro-CT images demonstrated that OVX-induced bone loss was prevented by HSYA treatment. bQuantitative analyses of parameters regarding to bone architecture, including BMD, BV/TV, Tb. N, Tb. Th, Tb. Sp. ${ }^{*} \mathrm{P}<0.05$, compared with Sham group; @P<0.05, compared with HSYA $(2.5 \mathrm{mg} / \mathrm{kg})$ group 

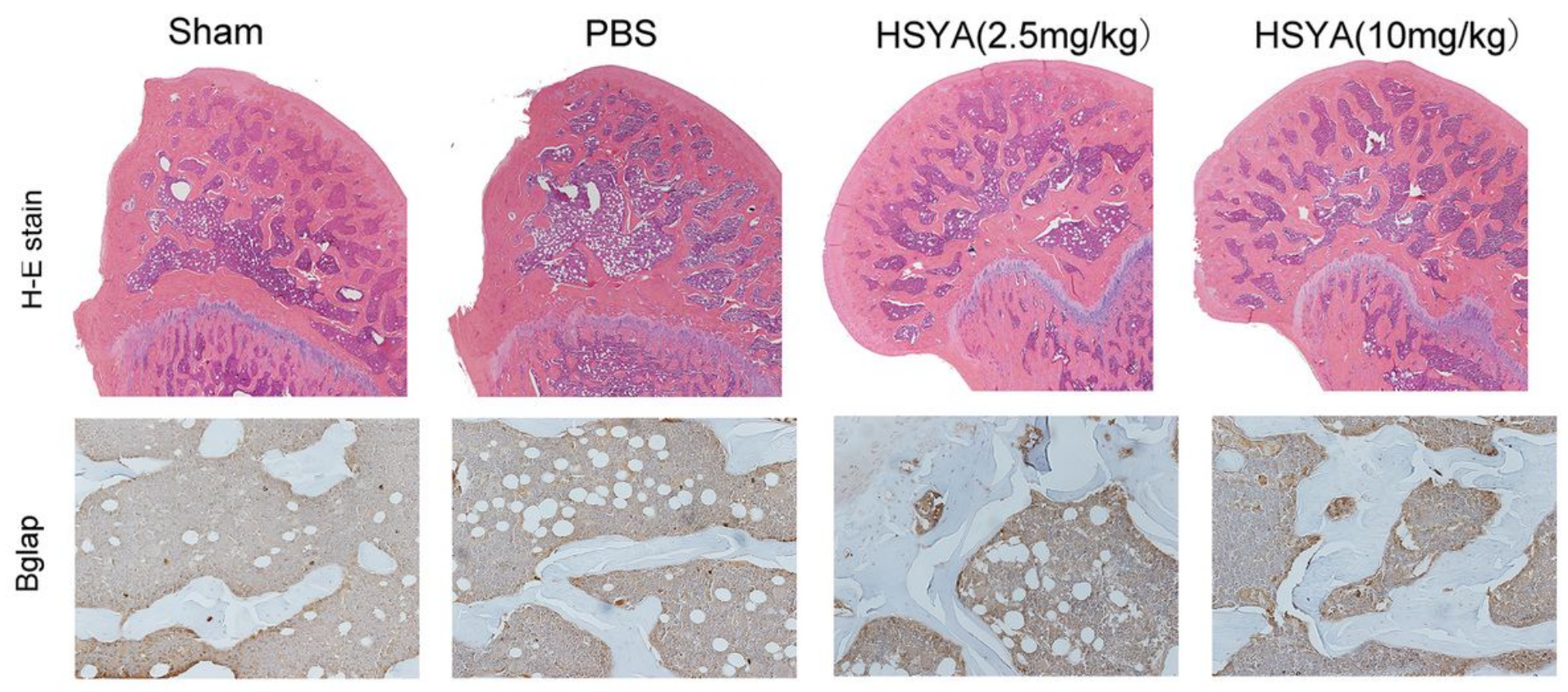

\section{Figure 8}

HSYA improved the trabecular structure and stimulated the expression of the Bglap in OVX mice. Representative images of $\mathrm{HE}$ and immunohistochemical staining of distal femoral trabecular bone. 


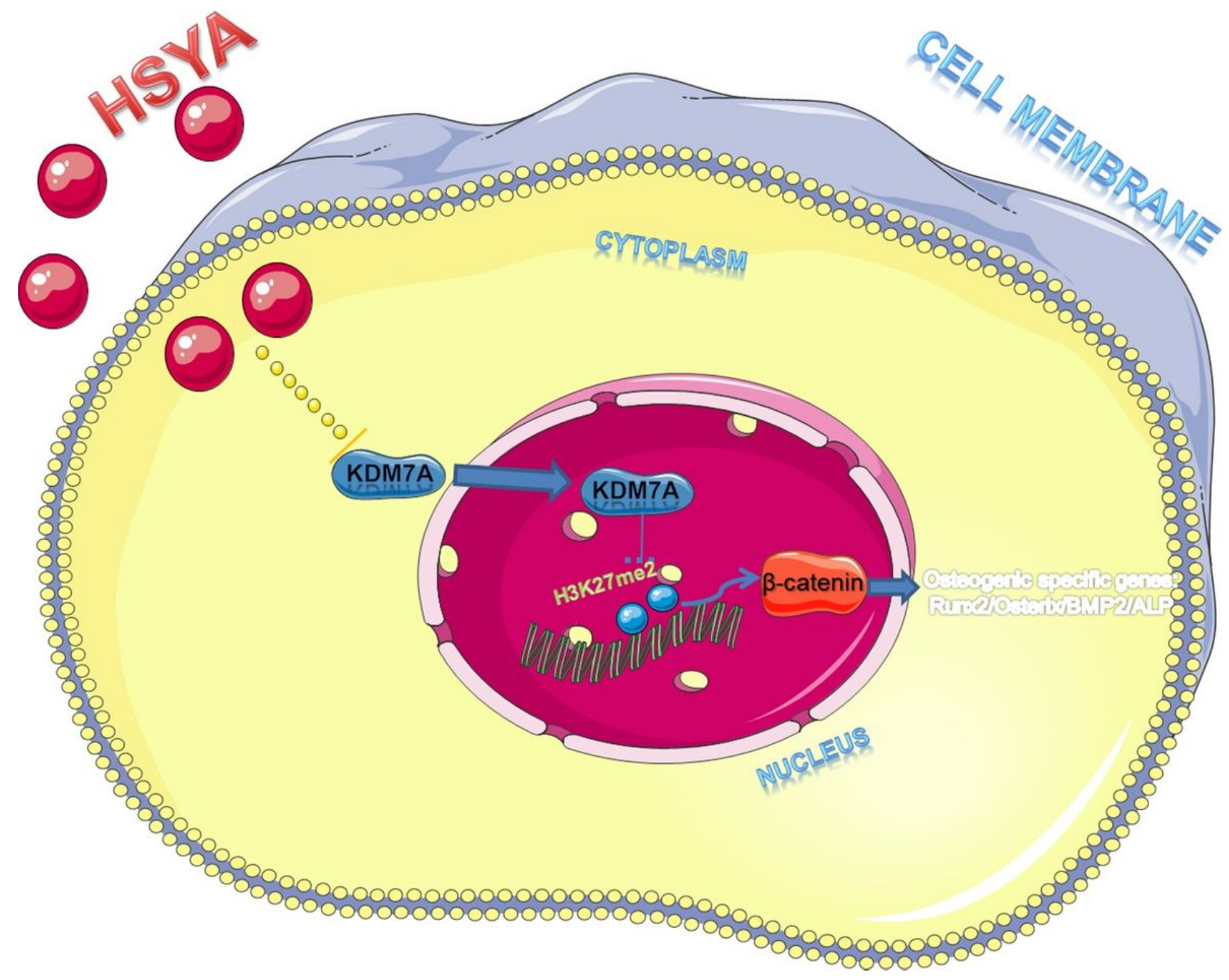

Figure 9

A potential working model for the promotion of HSYA on osteogenesis of hBMSCs. Our results indicated that HSYA might can enhance osteoblastic ability of hBMSCs via motivating KDM7A (demethylates H3K27me2)to activate $\beta$-catenin, which connects the Tcf/Lef family of transcription factors to regulate the expression of a series of downstream target genes through activation of canonical Wnt signaling, which promotes osteogenesis in hBMSCs.

\section{Supplementary Files}

This is a list of supplementary files associated with this preprint. Click to download.

- Supplementaryfile2.txt

- Supplementaryfile1.xls 